\title{
The Prolonged Minima and Maxima of Solar Activity
}

\author{
V.N. Dermendijev¹, G.T. Buyukliev¹, Y.Y. Shopov² \\ ${ }^{1}$ Department of Astronomy and NAO, 72 Lenin Blvd., 1784 Sofia, \\ Bulgaria \\ ${ }^{2}$ Faculty of Physics, University of Sofia, 5 Anton Ivanov Blvd., Sofia, \\ Bulgaria
}

\begin{abstract}
We study a new indirect index of solar activity - the Intensity of Luminescence of Cave Flowstone Microzones. This index correlates directly with the solar activity. Using a time series of the index with resolution 4-5 pixels/year we study some of the statistical properties of the solar activity cycle during 28 prolonged minima and 21 prolonged maxima.
\end{abstract}

\section{The new index of solar activity}

Proxy data on past solar activity nowadays provide a useful means of approaching the problem of solar magnetic field generation (Dermendjiev et al., 1990). For this purpose, however, we need time series of data capable of distinguishing individual solar cycles. In this work we study from this point of view 28 prolonged minima of solar activity in the past (until 22000 B.C.) using time series of a new indirect index.

The new index was named "Microzonality of Luminescence of Cave Flowstones" (Shopov and Dermendjiev, 1990). For its detection the method of Laser Luminescence Analysis (LLMZA) was developed (Shopov, 1987). If the growth rate of the flowstone is known (e.g. from the nuclear dating of the sample), the intensity curve of the luminescence, depending on the distance from the flowstone surface, can be transformed into a time series.

In our study we use time series with resolution of 4-5 pixels per year. They make it possible to obtain information on the phase, period and epoch of the minimum, and other data about the properties of the separate 11-year cycles. 


\section{The prolonged minima and maxima of the LLMZA data}

We had the possibility of obtaining time series of LLMZA data with time resolution about 5 pixels/year spanning 22000 years. It contains 110000 measurements. We use this long time series of proxy data of solar activity to seek answers to the following questions: 1) Does the 11-year cycle continue during the Maunder, Spörer, and other prolonged minima of the past solar activity? 2) Is the 11-year cycle typical of the epochs of normal activity and prolonged maxima of solar activity? and 3) Is there any correlation between duration of the minima, considered as phase shifts of a very long cycle reflecting possible global stochastic nature of solar activity, and the amplitude change of the subsequent activity?

We determined 28 prolonged minima and 21 prolonged maxima. Among them are the Modern and Medieval maxima, Maunder and Spörer minima, and the other 14 prolonged minima and maxima which have been found in ${ }^{14} \mathrm{C}$ time series. We study these peculiar intervals in the past solar activity by means of power spectral analysis.

The largest period for the Maunder minimum is 5.6 years and for the Spörer minimum 7.6 years. The power spectra for these two epochs differ insignificantly but they are rather different in comparison with the power spectra of the Medieval maximum.

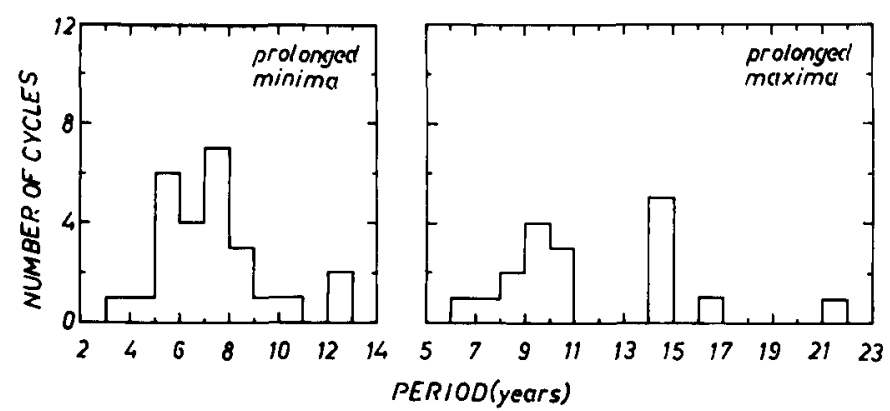

Fig. 1. Empirical distribution of the solar cycle periods for the prolonged minima and maxima.

The empirical distributions of the periods obtained for the prolonged minima and maxima determined from LLMZA data are shown on Fig. 1. As one can deduce from this figure, the solar cycle is shorter during the prolonged minima (mean value $T=7.85$ years). The distribution of the periods during the prolonged maxima shows a special feature. When the solar cycle is longer, the preferred period is 1415 years. It is interesting to note also that there are no periods of 11-14 years. The very good time resolution of LLMZA data also gives us the possibility of studying the correlation between the duration of the prolonged minima, considered as phase shifts of a very long cycle, and the amplitude change of the subsequent activity maxima. The results obtained from a 12000 years long time series are shown on 
Fig. 2. The correlation coefficient $r=0.422$ is positive and statistically significant. The data can be approximated by linear regressions.

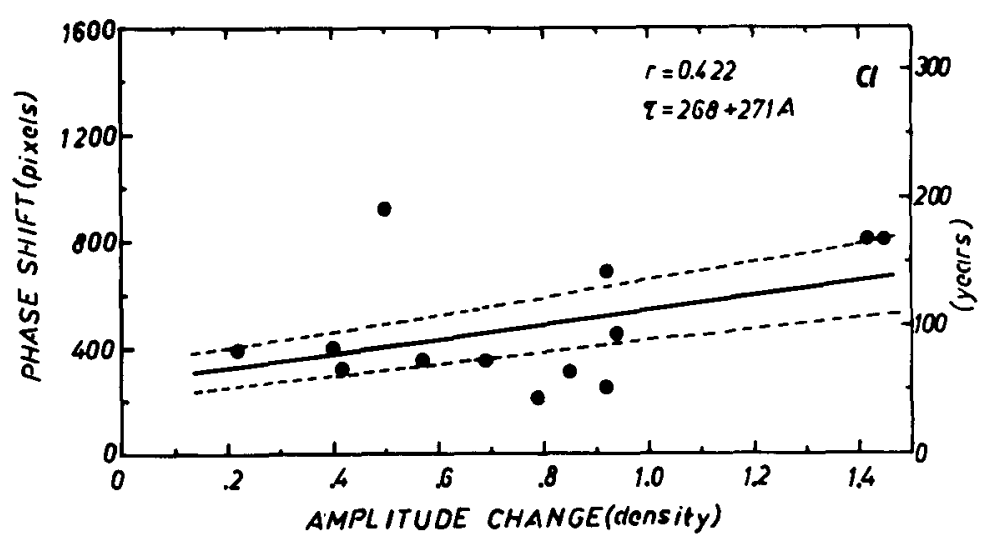

Fig. 2. Correlation diagram of the duration of the prolonged minima and amplitude change of the subsequent activity maxima for the time intervals $1985 \mathrm{AD}-10000 \mathrm{BC}$.

The problem of the nature of prolonged minima of solar activity is still under discussion. From a modern point of view the prolonged minima are considered as strange attractors (Ruzmaikin, 1985). For large dynamo numbers the solutions of the Lorenz system for the magnetic field are chaotic in time with episodes of very low amplitude. It will be interesting to discover if such a positive correlation exists between episodes of reduced activity and the amplitude of the envelopes of the magnetic field taken from theoretical models of nonlinear dynamos.

Acknowledgement. This work was supported by the Ministry of Culture, Science and Education under Grant No. 1162/88.

\section{References}

Dermendjiev, V.N., Shopov, Y.Y., Buyukliev, G.T.: 1990, Solar Phys. 128, 217

Ruzmaikin, A.A.: 1985, Solar Phys. 100, 125

Shopov, Y.Y.: 1987, in "Proc. of Int Symp. on Complex Research of Mountain Karst", Tbilisi, p. 6

Shopov, Y.Y., Dermendjiev, V.N.: 1990, Comptes rendus de l'Academie Bulg. des Sci., in press 